\title{
Exact travelling wave solutions for some nonlinear $(N+1)$-dimensional evolution equations
}

\author{
JONU LEE and RATHINASAMY SAKTHIVEL* \\ Department of Mathematics, Sungkyunkwan University, \\ Suwon 440-746, Republic of Korea \\ E-mails: jonulee@gmail.com / krsakthivel@yahoo.com
}

\begin{abstract}
In this paper, we implement the tanh-coth function method to construct the travelling wave solutions for $(N+1)$-dimensional nonlinear evolution equations. Four models, namely the $(N+1)$-dimensional generalized Boussinesq equation, $(N+1)$-dimensional sine-cosineGordon equation, $(N+1)$-double sinh-Gordon equation and $(N+1)$-sinh-cosinh-Gordon equation, are used as vehicles to conduct the analysis. These equations play a very important role in mathematical physics and engineering sciences. The implemented algorithm is quite efficient and is practically well suited for these problems. The computer symbolic systems such as Maple and Mathematica allow us to perform complicated and tedious calculations.
\end{abstract}

Mathematical subject classification: $35 \mathrm{~K} 58,35 \mathrm{C} 06,35 \mathrm{~A} 25$.

Key words: Nonlinear evolution equations, Travelling wave solutions, tanh-coth function method.

\section{Introduction}

It is well known that nonlinear evolution equations arise in number of scientific models including the propagation of shallow water waves, hydrodynamic, plasma physics, fluid mechanics, solid state physics, chemical kinematics, chemical chemistry, optical fiber and geochemistry. The investigation of travelling wave solutions of nonlinear evolution equations have been widely studied due 
to the significant applications in mathematical theory and other practical fields. A variety of powerful and direct methods have been proposed to obtain exact solutions of nonlinear evolution equations such as exp-function method $[1,11,12,18,20,26]$, Homotopy perturbation method [3, 4], variational iteration method [5, 15], Hirota's method [24], auxiliary equation method [10], Jacobi elliptic function method [14], hyperbolic function method [2] and so on.

However, practically there is no unified method that can be used to handle all types of nonlinearity. Another important method used to obtain exact solutions of nonlinear problem is the tanh function method [16]. The basic tanh-function method was introduced in the early 1990s (see [8,23] and references therein). The tanh function method with the help of Matlab or Mathematica is simple and effective, and it has been successfully applied to many kinds of nonlinear evolution equations $[8,9,13,27]$ for finding generalized solitary wave solutions and periodic solutions. Since then the method has been extended and generalized, the motivation being to obtain more solutions than are delivered by the basic tanhfunction method. Recently, El-Wakil et al. [6, 7] proposed a modified tanh-coth function method for constructing soliton and periodic solutions of nonlinear problems. Subsequently, Wazzan [22] used modified tanh-coth function method and obtained new solutions for some important nonlinear partial differential equations. More recently, Lee and Sakthivel [19] implemented modified tanhcoth function method for obtaining travelling wave solutions of two dimensional coupled Burger's equations.

Further, in recent years, much attention has been paid on the study of solutions of nonlinear wave equations in low dimensions. But there is little work on the high dimensional equations. Motivated by this consideration, in this paper we obtain the travelling wave solutions of high dimensional equations. The $(N+1)$-dimensional equations such as generalized Boussinesq equation, sinecosine-Gordon equation, double sinh-Gordon equation and sinh-cosinh-Gordon equation have significant applications in real world problems [21, 25]. Therefore, the search for exact solutions of these equations is of great importance and interest. The purpose of this paper is to obtain new travelling wave solutions of these $(N+1)$-dimensional equations by applying the tanh-coth function method. 


\section{Modified tanh-coth function method}

In this section, we will present briefly the modified tanh-coth function method in its systematized form $[6,7]$. Suppose we are given a nonlinear evolution equation in the form of a partial differential equation (PDE) for a function $u(t, x)$. First, we seek travelling wave solutions by taking $u(x, t)=u(\eta), \eta=k x-\omega t$, where $k$ and $\omega$ represent the wave number and velocity of the travelling wave respectively. Substitution into the PDE yields an ordinary differential equation (ODE) for $u(\eta)$. The ordinary differential equation is then integrated as long as all terms contain derivatives, where the integration constants are considered as zeros. The resulting ODE is then solved by the tanh-coth method which admits the use of a finite series of functions of the form

$$
u(\eta)=a_{0}+\sum_{n=1}^{M} a_{n} Y^{n}(\eta)+\sum_{n=1}^{M} b_{n} Y^{-n}(\eta),
$$

and the Riccati equation

$$
Y^{\prime}=A+C Y^{2}
$$

where $A$ and $C$ are constants to be prescribed later. Here $M$ is a positive integer that will be determined. The parameter $M$ is usually obtained by balancing the linear terms of highest order in the resulting equation with the highest order nonlinear terms. Substituting (1) in the ODE and using (2) results in an algebraic system of equations in powers of $Y$ that will lead to the determination of the parameters $a_{n}, b_{n}(n=0, \ldots, M), k$ and $\omega$.

In this paper, we will consider the following special solutions of the Riccati equation (2)

(i) $A=\frac{1}{2}, C=-\frac{1}{2}$, Eq. (2) has solutions $Y=\tanh \eta \pm i \operatorname{sech} \eta$ and $Y=$ $\operatorname{coth} \eta \pm \operatorname{csch} \eta$.

(ii) $A=1, C=-4$, Eq. (2) has solutions $Y=\frac{1}{2} \tanh 2 \eta$ and $Y=\frac{1}{4}(\tanh \eta+$ $\operatorname{coth} \eta$ ).

(iii) $A=1, C=4$, Eq. (2) has solutions $Y=\frac{1}{2} \tan 2 \eta$ and $Y=\frac{1}{4}(\tan \eta-$ $\cot \eta$ ). 


\section{Solution of $(N+1)$-dimensional generalized Boussinesq equation}

In this section, modified tanh-coth method will be applied to handle the $(N+$ 1)-dimensional generalized Boussinesq equation. The $(N+1)$-dimensional generalized Boussinesq equation is given by [25]

$$
u_{t t}=u_{x x}+\lambda\left(u^{n}\right)_{x x}+u_{x x x x}+\sum_{j=1}^{N-1} u_{y_{j} y_{j}}
$$

where $\lambda \neq 0$ is constant and $N>1$ is an integer. To solve the Eq. (3), consider the wave transformation

$$
u\left(x, y_{1}, y_{2}, \ldots, y_{N-1}, t\right)=u(\eta), \eta=\tau\left(x+\sum_{j=1}^{N-1} y_{j}-c t\right),
$$

here $\tau \neq 0$ and $c \neq 0$ are constants. Now the Eq. (3) can be written as

$$
\left(N-c^{2}\right) u^{\prime \prime}+\lambda\left(u^{n}\right)^{\prime \prime}+\tau^{2} u^{\prime \prime \prime \prime}=0,
$$

where the prime denotes derivative with respect to $\eta$. Integrating Eq. (4) with respect to $\eta$ and ignoring the constant of integration, we obtain

$$
\left(N-c^{2}\right) u^{\prime}+\lambda\left(u^{n}\right)^{\prime}+\tau^{2} u^{\prime \prime \prime}=0 .
$$

Next, we introduce the transformation $u^{n-1}=v$, then we have

$$
\begin{aligned}
u^{\prime}= & \frac{1}{n-1} v^{\frac{1}{n-1}-1} v^{\prime}, \quad\left(u^{n}\right)^{\prime}=\frac{n}{n-1} v^{\frac{1}{n-1}} v^{\prime}, \\
u^{\prime \prime \prime}= & \frac{(n-2)(2 n-3)}{(n-1)^{3}} v^{\frac{1}{n-1}-3}\left(v^{\prime}\right)^{3}+\frac{3(2-n)}{(n-1)^{2}} v^{\frac{1}{n-1}-2} v^{\prime} v^{\prime \prime} \\
& +\frac{1}{n-1} v^{\frac{1}{n-1}-1} v^{\prime \prime \prime} .
\end{aligned}
$$

Substituting these transformations (6) and (7) in Eq. (5), we can rewrite the $(N+1)$-dimensional generalized Boussinesq Eq. (3) in the following nonlinear ordinary differential equation of the form

$$
\begin{gathered}
\left(N-c^{2}\right)(n-1)^{2} v^{2} v^{\prime}+\lambda n(n-1)^{2} v^{3} v^{\prime}+\tau^{2}(n-2)(2 n-3)\left(v^{\prime}\right)^{3} \\
+3 \tau^{2}(n-1)(2-n) v v^{\prime} v^{\prime \prime}+\tau^{2}(n-1)^{2} v^{2} v^{\prime \prime \prime}=0 .
\end{gathered}
$$


To determine the parameter $M$, we balance the linear terms of highest order in Eq. (8) with the highest order nonlinear terms. This in turn gives $M=2$. As a result, the modified tanh-coth method (1) admits the use of the finite expansion

$$
v(\eta)=a_{0}+a_{1} Y+a_{2} Y^{2}+\frac{b_{1}}{Y}+\frac{b_{2}}{Y^{2}} .
$$

Substituting Eq. (9) in the reduced ODE (8) and using Eq. (2) collecting the coefficients of $Y$, yields a system of algebraic equations for $a_{0}, a_{1}, a_{2}, b_{1}, b_{2}, \tau$ and $c$.

Case (I): If we set $A=\frac{1}{2}$ and $C=-\frac{1}{2}$ in equation (2), and solving the system of algebraic equations using Maple, we obtain the following three sets of nontrivial solutions:

$$
\begin{aligned}
& \left\{a_{0}=\frac{(n+1)\left(c^{2}-N\right)}{2 \lambda}, a_{1}=0, a_{2}=-\frac{(n+1)\left(c^{2}-N\right)}{2 \lambda},\right. \\
& \left.b_{1}=0, b_{2}=0, \tau= \pm(n-1) \sqrt{c^{2}-N}, c=c\right\}, \\
& \left\{a_{0}=\frac{(n+1)\left(c^{2}-N\right)}{2 \lambda}, a_{1}=0, a_{2}=0,\right. \\
& \left.b_{1}=0, b_{2}=-\frac{(n+1)\left(c^{2}-N\right)}{2 \lambda}, \tau= \pm(n-1) \sqrt{c^{2}-N}, c=c\right\}, \\
& \left\{a_{0}=\frac{(n+1)\left(c^{2}-N\right)}{4 \lambda}, a_{1}=0, a_{2}=-\frac{(n+1)\left(c^{2}-N\right)}{8 \lambda},\right. \\
& \left.b_{1}=0, b_{2}=-\frac{(n+1)\left(c^{2}-N\right)}{8 \lambda}, \tau= \pm \frac{(n-1) \sqrt{c^{2}-N}}{2}, c=c\right\} .
\end{aligned}
$$

Substituting $Y=\tanh \eta \pm i \operatorname{sech} \eta$ and $Y=\operatorname{coth} \eta \pm \operatorname{csch} \eta$ in equation (9), the first two sets (10) and (11) gives the wave solutions

$$
\begin{aligned}
& u_{1,1}(x, \tilde{y}, t)=\left[\frac{(n+1)\left(c^{2}-N\right)}{2 \lambda}\left\{1-(\tanh \eta \pm i \operatorname{sech} \eta)^{ \pm 2}\right\}\right]^{\frac{1}{n-1}}, \\
& u_{1,2}(x, \tilde{y}, t)=\left[\frac{(n+1)\left(c^{2}-N\right)}{2 \lambda}\left\{1-(\operatorname{coth} \eta \pm \operatorname{csch} \eta)^{ \pm 2}\right\}\right]^{\frac{1}{n-1}},
\end{aligned}
$$


where $\tilde{y}=y_{1}, y_{2}, \cdots, y_{N-1}$ and $\eta= \pm(n-1) \sqrt{c^{2}-N}\left(x+\sum_{j=1}^{N-1} y_{j}-c t\right)$. Finally, the third set gives the wave solutions

$$
\begin{aligned}
u_{1,3}(x, \tilde{y}, t)= & {\left[\frac { ( n + 1 ) ( c ^ { 2 } - N ) } { 8 \lambda } \left\{2-(\tanh \eta \pm i \operatorname{sech} \eta)^{2}\right.\right.} \\
& \left.\left.-\frac{1}{(\tanh \eta \pm i \operatorname{sech} \eta)^{2}}\right\}\right]^{\frac{1}{n-1}}, \\
u_{1,4}(x, \tilde{y}, t)= & {\left[\frac { ( n + 1 ) ( c ^ { 2 } - N ) } { 8 \lambda } \left\{2-(\operatorname{coth} \eta \pm \operatorname{csch} \eta)^{2}\right.\right.} \\
& \left.\left.-\frac{1}{(\operatorname{coth} \eta \pm \operatorname{csch} \eta)^{2}}\right\}\right]^{\frac{1}{n-1}},
\end{aligned}
$$

where

$$
\eta= \pm \frac{(n-1) \sqrt{c^{2}-N}}{2}\left(x+\sum_{j=1}^{N-1} y_{j}-c t\right)
$$

Case (II): If we set $A=1$ and $C=-4$ in equation (2) and by the same calculation as above, the following sets of nontrivial solutions are obtained:

$$
\begin{aligned}
& \left\{a_{0}=\frac{(n+1)\left(c^{2}-N\right)}{2 \lambda}, a_{1}=0, a_{2}=-\frac{2(n+1)\left(c^{2}-N\right)}{\lambda},\right. \\
& \left.b_{1}=0, b_{2}=0, \tau= \pm \frac{(n-1) \sqrt{c^{2}-N}}{4}, c=c\right\}, \\
& \left\{a_{0}=\frac{(n+1)\left(c^{2}-N\right)}{2 \lambda}, a_{1}=0, a_{2}=0,\right. \\
& \left.b_{1}=0, b_{2}=-\frac{(n+1)\left(c^{2}-N\right)}{8 \lambda}, \tau= \pm \frac{(n-1) \sqrt{c^{2}-N}}{4}, c=c\right\}, \\
& \left\{a_{0}=\frac{(n+1)\left(c^{2}-N\right)}{4 \lambda}, a_{1}=0, a_{2}=-\frac{(n+1)\left(c^{2}-N\right)}{2 \lambda},\right. \\
& \left.b_{1}=0, b_{2}=-\frac{(n+1)\left(c^{2}-N\right)}{32 \lambda}, \tau= \pm \frac{(n-1) \sqrt{c^{2}-N}}{8}, c=c\right\} .
\end{aligned}
$$


The first two sets gives the following wave solutions for Eq. (3):

$$
\begin{aligned}
& u_{1,5}(x, \tilde{y}, t)=\left[\frac{(n+1)\left(c^{2}-N\right)}{2 \lambda}\left\{1-(\tanh 2 \eta)^{ \pm 2}\right\}\right]^{\frac{1}{n-1}}, \\
& u_{1,6}(x, \tilde{y}, t)=\left[\frac{(n+1)\left(c^{2}-N\right)}{2 \lambda}\left\{1-\left(\frac{\tanh +\operatorname{coth} \eta}{2}\right)^{ \pm 2}\right\}\right]^{\frac{1}{n-1}},
\end{aligned}
$$

where $\eta= \pm \frac{(n-1) \sqrt{c^{2}-N}}{4}\left(x+\sum_{j=1}^{N-1} y_{j}-c t\right)$. The third set gives the solutions

$$
\begin{aligned}
u_{1,7}(x, \tilde{y}, t)= & {\left[\frac{(n+1)\left(c^{2}-N\right)}{8 \lambda}\left\{2-\tanh ^{2} 2 \eta-\operatorname{coth}^{2} 2 \eta\right\}\right]^{\frac{1}{n-1}}, } \\
u_{1,8}(x, \tilde{y}, t)= & {\left[\frac { ( n + 1 ) ( c ^ { 2 } - N ) } { 3 2 \lambda } \left\{8-(\tanh \eta+\operatorname{coth} \eta)^{2}\right.\right.} \\
& \left.\left.-\frac{16}{(\tanh \eta+\operatorname{coth} \eta)^{2}}\right\}\right]^{\frac{1}{n-1}}
\end{aligned}
$$

where

$$
\eta= \pm \frac{(n-1) \sqrt{c^{2}-N}}{8}\left(x+\sum_{j=1}^{N-1} y_{j}-c t\right)
$$

Case (III): If we set $A=1$ and $C=4$ in Eq. (2), and solving the system of algebraic equations using Maple, we get the following sets of solutions:

$$
\begin{aligned}
& \left\{a_{0}=\frac{(n+1)\left(c^{2}-N\right)}{2 \lambda}, a_{1}=0, a_{2}=\frac{2(n+1)\left(c^{2}-N\right)}{\lambda},\right. \\
& \left.b_{1}=0, b_{2}=0, \tau= \pm \frac{(n-1) \sqrt{N-c^{2}}}{4}, c=c\right\} \\
& \left\{a_{0}=\frac{(n+1)\left(c^{2}-N\right)}{2 \lambda}, a_{1}=0, a_{2}=0,\right. \\
& \left.b_{1}=0, b_{2}=\frac{(n+1)\left(c^{2}-N\right)}{8 \lambda}, \tau= \pm \frac{(n-1) \sqrt{N-c^{2}}}{4}, c=c\right\},
\end{aligned}
$$




$$
\begin{aligned}
& \left\{a_{0}=\frac{(n+1)\left(c^{2}-N\right)}{4 \lambda}, a_{1}=0, a_{2}=\frac{(n+1)\left(c^{2}-N\right)}{2 \lambda},\right. \\
& \left.b_{1}=0, b_{2}=\frac{(n+1)\left(c^{2}-N\right)}{32 \lambda}, \tau= \pm \frac{(n-1) \sqrt{N-c^{2}}}{8}, c=c\right\} .
\end{aligned}
$$

By the same calculation as above, we obtain the following wave solutions of Eq. (3) from the first two sets of equations (24) and (25)

$$
\begin{gathered}
u_{1,9}(x, \tilde{y}, t)=\left[\frac{(n+1)\left(c^{2}-N\right)}{2 \lambda}\left\{1+(\tan 2 \eta)^{ \pm 2}\right\}\right]^{\frac{1}{n-1}}, \\
u_{1,10}(x, \tilde{y}, t)=\left[\frac{(n+1)\left(c^{2}-N\right)}{2 \lambda}\left\{1-\left(\frac{\tan \eta-\cot \eta}{2}\right)^{ \pm 2}\right\}\right]^{\frac{1}{n-1}},
\end{gathered}
$$

where $\eta= \pm \frac{(n-1) \sqrt{N-c^{2}}}{4}\left(x+\sum_{j=1}^{N-1} y_{j}-c t\right)$. Finally, the third set (26) gives the wave solutions

$$
\begin{aligned}
u_{1,11}(x, \tilde{y}, t)= & {\left[\frac{(n+1)\left(c^{2}-N\right)}{8 \lambda}\left\{2+\tan ^{2} 2 \eta+\cot ^{2} 2 \eta\right\}\right]^{\frac{1}{n-1}}, } \\
u_{1,12}(x, \tilde{y}, t)= & {\left[\frac { ( n + 1 ) ( c ^ { 2 } - N ) } { 3 2 \lambda } \left\{8+(\tan \eta-\cot \eta)^{2}\right.\right.} \\
& \left.\left.+\frac{16}{(\tan \eta-\cot \eta)^{2}}\right\}\right]^{\frac{1}{n-1}}
\end{aligned}
$$

where

$$
\eta= \pm \frac{(n-1) \sqrt{N-c^{2}}}{8}\left(x+\sum_{j=1}^{N-1} y_{j}-c t\right)
$$

\section{Solution of $(N+1)$-dimensional sine-cosine-Gordon equation}

The $(N+1)$-dimensional sine-cosine-Gordon equation is given by [21]

$$
\sum_{j=1}^{N} u_{x_{j} x_{j}}-u_{t t}-\alpha \cos (u)-\beta \sin (2 u)=0 .
$$


Consider the wave transformation

$$
u\left(x_{1}, x_{2}, \ldots, x_{N}, t\right)=u(\eta), \eta=\tau\left(\sum_{j=1}^{N} x_{j}-c t\right),
$$

where $\tau \neq 0$ and $c \neq 0$ are constants. With the help of the transformation, Eq. (31) can be written as

$$
\tau^{2}\left(N-c^{2}\right) u^{\prime \prime}-\alpha \cos (u)-\beta \sin (2 u)=0,
$$

where the prime denotes derivative with respect to $\eta$. Now, we introduce the transformation $u=2 \tan ^{-1} v$, then we have

$$
u^{\prime \prime}=\frac{2\left(v^{\prime \prime}+v^{\prime \prime} v^{2}-2\left(v^{\prime}\right)^{2} v\right)}{\left(1+v^{2}\right)^{2}}, \quad \cos (u)=\frac{1-v^{2}}{1+v^{2}}, \quad \sin (2 u)=\frac{4 v\left(1-v^{2}\right)}{\left(1+v^{2}\right)^{2}} .
$$

Substituting these transformations (33) in Eq. (32), we can rewrite the $(N+1)$ dimensional sine-cosine-Gordon Eq. (31) in the following form

$$
2 \tau^{2}\left(N-c^{2}\right)\left(1+v^{2}\right) v^{\prime \prime}-4 \tau^{2}\left(N-c^{2}\right) v\left(v^{\prime}\right)^{2}+\left(v^{2}-1\right)\left(\alpha v^{2}+4 \beta v+\alpha\right)=0 .
$$

To determine the parameter $M$, we balance the linear terms of highest order in Eq. (34) with the highest order nonlinear terms which gives $M=2$. As a result, the modified tanh-coth method (1) admits the use of the finite expansion

$$
v(\eta)=a_{0}+a_{1} Y+a_{2} Y^{2}+\frac{b_{1}}{Y}+\frac{b_{2}}{Y^{2}} .
$$

Substituting Eq. (35) in the reduced ODE (34) and using Eq. (2) collecting the coefficients of $Y$, yields a system of algebraic equations for $a_{0}, a_{1}, a_{2}, b_{1}, b_{2}, \tau$ and $c$.

Case (I): If we set $A=\frac{1}{2}$ and $C=-\frac{1}{2}$ in equation (2), and solving the system of algebraic equations using Maple, we obtain the following three sets of solutions:

$$
\begin{aligned}
& \left\{a_{0}=-\frac{2 \beta}{\alpha}, a_{1}= \pm \frac{\sqrt{4 \beta^{2}-\alpha^{2}}}{\alpha}, a_{2}=0,\right. \\
& \left.b_{1}=0, b_{2}=0, \tau=\tau, c= \pm \frac{\sqrt{\alpha^{2}-4 \beta^{2}+2 \tau^{2} N \beta}}{\tau \sqrt{2 \beta}}\right\},
\end{aligned}
$$




$$
\begin{aligned}
& \left\{a_{0}=-\frac{2 \beta}{\alpha}, a_{1}=0, a_{2}=0,\right. \\
& \left.b_{1}= \pm \frac{\sqrt{4 \beta^{2}-\alpha^{2}}}{\alpha}, b_{2}=0, \tau=\tau, c= \pm \frac{\sqrt{\alpha^{2}-4 \beta^{2}+2 \tau^{2} N \beta}}{\tau \sqrt{2 \beta}}\right\}, \\
& \left\{a_{0}=-\frac{2 \beta}{\alpha}, a_{1}= \pm \frac{\sqrt{4 \beta^{2}-\alpha^{2}}}{2 \alpha}, a_{2}=0, b_{1}= \pm \frac{\sqrt{4 \beta^{2}-\alpha^{2}}}{2 \alpha},\right. \\
& \left.b_{2}=0, \tau=\tau, c= \pm \frac{\sqrt{\alpha^{2}-4 \beta^{2}+8 \tau^{2} N \beta}}{2 \tau \sqrt{2 \beta}}\right\} .
\end{aligned}
$$

Substituting $Y=\tanh \eta \pm i \operatorname{sech} \eta$ and $Y=\operatorname{coth} \eta \pm \operatorname{csch} \eta$ in equation (35), the first two sets (36) and (37) gives the wave solutions

$$
\begin{aligned}
& u_{2,1}(\tilde{x}, t)=2 \tan ^{-1}\left[-\frac{2 \beta}{\alpha} \pm \frac{\sqrt{4 \beta^{2}-\alpha^{2}}}{\alpha}(\tanh \eta \pm i \operatorname{sech} \eta)^{ \pm 1}\right], \\
& u_{2,2}(\tilde{x}, t)=2 \tan ^{-1}\left[-\frac{2 \beta}{\alpha} \pm \frac{\sqrt{4 \beta^{2}-\alpha^{2}}}{\alpha}(\operatorname{coth} \eta \pm \operatorname{csch} \eta)^{ \pm 1}\right],
\end{aligned}
$$

where $\tilde{x}=x_{1}, x_{2}, \ldots, x_{N}$ and $\eta=\tau\left(\sum_{j=1}^{N} x_{j} \pm \frac{\sqrt{\alpha^{2}-4 \beta^{2}+2 \tau^{2} N \beta}}{\tau \sqrt{2 \beta}} t\right)$. Finally, the third set gives the wave solutions

$$
\begin{aligned}
u_{2,3}(\widetilde{x}, t)= & 2 \tan ^{-1}\left[-\frac{2 \beta}{\alpha} \pm \frac{\sqrt{4 \beta^{2}-\alpha^{2}}}{2 \alpha}\{(\tanh \eta \pm i \operatorname{sech} \eta)\right. \\
& \left.\left.+\frac{1}{(\tanh \eta \pm i \operatorname{sech} \eta)}\right\}\right], \\
u_{2,4}(\tilde{x}, t)= & 2 \tan ^{-1}\left[-\frac{2 \beta}{\alpha} \pm \frac{\sqrt{4 \beta^{2}-\alpha^{2}}}{2 \alpha}\{(\operatorname{coth} \eta \pm \operatorname{csch} \eta)\right. \\
& \left.\left.+\frac{1}{(\operatorname{coth} \eta \pm \operatorname{csch} \eta)}\right\}\right]
\end{aligned}
$$

where

$$
\eta=\tau\left(\sum_{j=1}^{N} x_{j} \pm \frac{\sqrt{\alpha^{2}-4 \beta^{2}+8 \tau^{2} N \beta}}{2 \tau \sqrt{2 \beta}} t\right) .
$$


Case (II): If we set $A=1$ and $C=-4$ in equation (2) and by the same calculation as above, the following sets of solutions are obtained:

$$
\begin{aligned}
& \left\{a_{0}=-\frac{2 \beta}{\alpha}, a_{1}= \pm \frac{2 \sqrt{4 \beta^{2}-\alpha^{2}}}{\alpha}, a_{2}=0,\right. \\
& \left.b_{1}=0, b_{2}=0, \tau=\tau, c= \pm \frac{\sqrt{\alpha^{2}-4 \beta^{2}+32 \tau^{2} N \beta}}{4 \tau \sqrt{2 \beta}}\right\}, \\
& \left\{a_{0}=-\frac{2 \beta}{\alpha}, a_{1}=0, a_{2}=0,\right. \\
& \left.b_{1}= \pm \frac{\sqrt{4 \beta^{2}-\alpha^{2}}}{2 \alpha}, b_{2}=0, \tau=\tau, c= \pm \frac{\sqrt{\alpha^{2}-4 \beta^{2}+32 \tau^{2} N \beta}}{4 \tau \sqrt{2 \beta}}\right\}, \\
& \left\{a_{0}=-\frac{2 \beta}{\alpha}, a_{1}= \pm \frac{\sqrt{4 \beta^{2}-\alpha^{2}}}{\alpha}, a_{2}=0,\right. \\
& \left.b_{1}= \pm \frac{\sqrt{4 \beta^{2}-\alpha^{2}}}{4 \alpha}, b_{2}=0, \tau=\tau, c= \pm \frac{\sqrt{\alpha^{2}-4 \beta^{2}+128 \tau^{2} N \beta}}{8 \tau \sqrt{2 \beta}}\right\} .
\end{aligned}
$$

The first two sets gives the following wave solutions of Eq. (31):

$$
\begin{aligned}
& u_{2,5}(\tilde{x}, t)=2 \tan ^{-1}\left[-\frac{2 \beta}{\alpha} \pm \frac{\sqrt{4 \beta^{2}-\alpha^{2}}}{\alpha}(\tanh 2 \eta)^{ \pm 1}\right], \\
& u_{2,6}(\tilde{x}, t)=2 \tan ^{-1}\left[-\frac{2 \beta}{\alpha} \pm \frac{\sqrt{4 \beta^{2}-\alpha^{2}}}{\alpha}(\tanh \eta+\operatorname{coth} \eta)^{ \pm 1}\right],
\end{aligned}
$$

where $\eta=\tau\left(\sum_{j=1}^{N} x_{j} \pm \frac{\sqrt{\alpha^{2}-4 \beta^{2}+32 \tau^{2} N \beta}}{4 \tau \sqrt{2 \beta}} t\right)$. The third set gives the wave solutions

$$
\begin{aligned}
u_{2,7}(\tilde{x}, t)= & 2 \tan ^{-1}\left[-\frac{2 \beta}{\alpha} \pm \frac{\sqrt{4 \beta^{2}-\alpha^{2}}}{2 \alpha}(\tanh 2 \eta+\operatorname{coth} 2 \eta)\right] \\
u_{2,8}(\tilde{x}, t)= & 2 \tan ^{-1}\left[-\frac{2 \beta}{\alpha} \pm \frac{\sqrt{4 \beta^{2}-\alpha^{2}}}{4 \alpha}\{(\tanh \eta+\operatorname{coth} \eta)\right. \\
& \left.\left.+\frac{4}{(\tanh \eta+\operatorname{coth} \eta)}\right\}\right]
\end{aligned}
$$


where

$$
\eta=\tau\left(\sum_{j=1}^{N} x_{j} \pm \frac{\sqrt{\alpha^{2}-4 \beta^{2}+128 \tau^{2} N \beta}}{8 \tau \sqrt{2 \beta}} t\right)
$$

Case (III): If we set $A=1$ and $C=4$ in Eq. (2), and solving the system of algebraic equations using Maple, we get the following sets of solutions:

$$
\begin{aligned}
& \left\{a_{0}=-\frac{2 \beta}{\alpha}, a_{1}= \pm \frac{2 \sqrt{\alpha^{2}-4 \beta^{2}}}{\alpha}, a_{2}=0,\right. \\
& \left.b_{1}=0, b_{2}=0, \tau= \pm \frac{\sqrt{\alpha^{2}-4 \beta^{2}}}{4 \sqrt{2 \beta\left(N-c^{2}\right)}}, c=c\right\}, \\
& \left\{a_{0}=-\frac{2 \beta}{\alpha}, a_{1}=0, a_{2}=0,\right. \\
& \left.b_{1}= \pm \frac{\sqrt{\alpha^{2}-4 \beta^{2}}}{2 \alpha}, b_{2}=0, \tau=\tau, c= \pm \frac{\sqrt{-\alpha^{2}+4 \beta^{2}+32 \tau^{2} N \beta}}{4 \tau \sqrt{2 \beta}}\right\}, \\
& \left\{a_{0}=-\frac{2 \beta}{\alpha}, a_{1}= \pm \frac{\sqrt{\alpha^{2}-4 \beta^{2}}}{\alpha}, a_{2}=0,\right. \\
& \left.b_{1}=\mp \frac{\sqrt{\alpha^{2}-4 \beta^{2}}}{4 \alpha}, b_{2}=0, \tau=\tau, c= \pm \frac{\sqrt{-\alpha^{2}+4 \beta^{2}+128 \tau^{2} N \beta}}{8 \tau \sqrt{2 \beta}}\right\} .
\end{aligned}
$$

By the same calculation as above, we obtain the following wave solutions of Eq. (31) from the first two sets of equations (50) and (51)

$$
\begin{aligned}
& u_{2,9}(\tilde{x}, t)=2 \tan ^{-1}\left[-\frac{2 \beta}{\alpha} \pm \frac{\sqrt{\alpha^{2}-4 \beta^{2}}}{\alpha}(\tan 2 \eta)^{ \pm 1}\right], \\
& u_{2,10}(\tilde{x}, t)=2 \tan ^{-1}\left[-\frac{2 \beta}{\alpha} \pm \frac{\sqrt{\alpha^{2}-4 \beta^{2}}}{\alpha}\left(\frac{\tan \eta-\cot \eta}{2}\right)^{ \pm 1}\right],
\end{aligned}
$$

where $\eta= \pm \frac{\sqrt{\alpha^{2}-4 \beta^{2}}}{4 \sqrt{2 \beta\left(N-c^{2}\right)}}\left(\sum_{j=1}^{N} x_{j}-c t\right)$. Finally, the third set (52) gives the wave solutions

$$
u_{2,11}(\tilde{x}, t)=2 \tan ^{-1}\left[-\frac{2 \beta}{\alpha} \pm \frac{\sqrt{\alpha^{2}-4 \beta^{2}}}{2 \alpha}(\tan 2 \eta-\cot 2 \eta)\right],
$$




$$
\begin{aligned}
u_{2,12}(\tilde{x}, t)= & 2 \tan ^{-1}\left[-\frac{2 \beta}{\alpha} \pm \frac{\sqrt{\alpha^{2}-4 \beta^{2}}}{2 \alpha}\left\{\frac{(\tan \eta-\cot \eta)}{2}\right.\right. \\
& \left.\left.-\frac{2}{(\tan \eta-\cot \eta)}\right\}\right],
\end{aligned}
$$

where

$$
\eta=\tau\left(\sum_{j=1}^{N} x_{j} \pm \frac{\sqrt{-\alpha^{2}+4 \beta^{2}+128 \tau^{2} N \beta}}{8 \tau \sqrt{2 \beta}} t\right) .
$$

\section{Solution of $(N+1)$-dimensional double sinh-Gordon equation}

In this section, consider the following $(N+1)$-dimensional double sinh-Gordon equation [21]

$$
\sum_{j=1}^{N} u_{x_{j} x_{j}}-u_{t t}-\alpha \sinh (u)-\beta \sinh (2 u)=0 .
$$

By using the transformation $u\left(x_{1}, x_{2}, \cdots, x_{N}, t\right)=u(\eta), \eta=\tau\left(\sum_{j=1}^{N} x_{j}-c t\right)$, here $\tau \neq 0$ and $c \neq 0$ are constants, the Eq. (57) can be written as

$$
\tau^{2}\left(N-c^{2}\right) u^{\prime \prime}-\alpha \sinh (u)-\beta \sinh (2 u)=0,
$$

where the prime denotes derivative with respect to $\eta$. Next we introduce the transformation $u=\ln v$, then we obtain

$$
u^{\prime \prime}=\frac{v^{\prime \prime} v-\left(v^{\prime}\right)^{2}}{v^{2}}, \quad \sinh (u)=\frac{v-v^{-1}}{2}, \quad \sinh (2 u)=\frac{v^{2}-v^{-2}}{2} .
$$

Substituting these transformations (59) in Eq. (58), we can rewrite the Eq. (57) in the following form

$$
2 \tau^{2}\left(N-c^{2}\right)\left(v^{\prime \prime} v-\left(v^{\prime}\right)^{2}\right)-\alpha\left(v^{3}-v\right)-\beta\left(v^{4}-1\right)=0 .
$$

To determine the parameter $M$, we balance the linear terms of highest order in Eq. (60) with the highest order nonlinear terms. This in turn gives $M=1$. As a result, the modified tanh-coth method (1) admits the use of the finite expansion

$$
v(\eta)=a_{0}+a_{1} Y+\frac{b_{1}}{Y} .
$$

Substituting Eq. (61) in the reduced ODE (60) and using Eq. (2) collecting the coefficients of $Y$, yields a system of algebraic equations for $a_{0}, a_{1}, b_{1}, \tau$ and $c$. 
Case (I): If we set $A=\frac{1}{2}$ and $C=-\frac{1}{2}$ in equation (2), and solving the system of algebraic equations using Maple, we obtain the following three sets of solutions:

$$
\begin{aligned}
& \left\{a_{0}=-\frac{\alpha}{2 \beta}, a_{1}= \pm \frac{\sqrt{\alpha^{2}-4 \beta^{2}}}{2 \beta}, b_{1}=0,\right. \\
& \left.\tau=\tau, c= \pm \frac{\sqrt{-\alpha^{2}+4 \beta^{2}+2 \tau^{2} N \beta}}{\tau \sqrt{2 \beta}}\right\}, \\
& \left\{a_{0}=-\frac{\alpha}{2 \beta}, a_{1}=0, b_{1}= \pm \frac{\sqrt{\alpha^{2}-4 \beta^{2}}}{2 \beta},\right. \\
& \left.\tau=\tau, c= \pm \frac{\sqrt{-\alpha^{2}+4 \beta^{2}+2 \tau^{2} N \beta}}{\tau \sqrt{2 \beta}}\right\}, \\
& \left\{a_{0}=-\frac{\alpha}{2 \beta}, a_{1}= \pm \frac{\sqrt{\alpha^{2}-4 \beta^{2}}}{4 \beta}, b_{1}= \pm \frac{\sqrt{\alpha^{2}-4 \beta^{2}}}{4 \beta},\right. \\
& \left.\tau=\tau, c= \pm \frac{\sqrt{-\alpha^{2}+4 \beta^{2}+8 \tau^{2} N \beta}}{2 \tau \sqrt{2 \beta}}\right\} .
\end{aligned}
$$

Substituting $Y=\tanh \eta \pm i \operatorname{sech} \eta$ and $Y=\operatorname{coth} \eta \pm \operatorname{csch} \eta$ in equation (61), the first two sets (62) and (63) gives the wave solutions

$$
\begin{aligned}
& u_{3,1}(\tilde{x}, t)=\ln \left[-\frac{\alpha}{2 \beta} \pm \frac{\sqrt{\alpha^{2}-4 \beta^{2}}}{2 \beta}(\tanh \eta \pm i \operatorname{sech} \eta)^{ \pm 1}\right], \\
& u_{3,2}(\tilde{x}, t)=\ln \left[-\frac{\alpha}{2 \beta} \pm \frac{\sqrt{\alpha^{2}-4 \beta^{2}}}{2 \beta}(\operatorname{coth} \eta \pm \operatorname{csch} \eta)^{ \pm 1}\right],
\end{aligned}
$$

where $\tilde{x}=x_{1}, x_{2}, \cdots, x_{N}$ and $\eta=\tau\left(\sum_{j=1}^{N} x_{j} \pm \frac{\sqrt{-\alpha^{2}+4 \beta^{2}+2 \tau^{2} N \beta}}{\tau \sqrt{2 \beta}} t\right)$. Finally, the third set gives the solutions

$$
\begin{aligned}
u_{3,3}(\tilde{x}, t)= & \ln \left[-\frac{\alpha}{2 \beta} \pm \frac{\sqrt{\alpha^{2}-4 \beta^{2}}}{4 \beta}\{(\tanh \eta \pm i \operatorname{sech} \eta)\right. \\
& \left.\left.+\frac{1}{(\tanh \eta \pm i \operatorname{sech} \eta)}\right\}\right]
\end{aligned}
$$




$$
\begin{aligned}
u_{3,4}(\tilde{x}, t)= & \ln \left[-\frac{\alpha}{2 \beta} \pm \frac{\sqrt{\alpha^{2}-4 \beta^{2}}}{4 \beta}\{(\operatorname{coth} \eta \pm \operatorname{csch} \eta)\right. \\
& \left.\left.+\frac{1}{(\operatorname{coth} \eta \pm \operatorname{csch} \eta)}\right\}\right]
\end{aligned}
$$

where

$$
\eta=\tau\left(\sum_{j=1}^{N} x_{j} \pm \frac{\sqrt{-\alpha^{2}+4 \beta^{2}+8 \tau^{2} N \beta}}{2 \tau \sqrt{2 \beta}} t\right)
$$

Case (II): If we set $A=1$ and $C=-4$ in equation (2) and by the same calculation as above, the following sets of solutions are obtained:

$$
\begin{aligned}
& \left\{a_{0}=-\frac{\alpha}{2 \beta}, a_{1}= \pm \frac{\sqrt{\alpha^{2}-4 \beta^{2}}}{\beta}, b_{1}=0,\right. \\
& \left.\tau= \pm \frac{\sqrt{\alpha^{2}-4 \beta^{2}}}{4 \sqrt{2 \beta\left(N-c^{2}\right)}}, c=c\right\}, \\
& \left\{a_{0}=-\frac{\alpha}{2 \beta}, a_{1}=0, b_{1}= \pm \frac{\sqrt{\alpha^{2}-4 \beta^{2}}}{4 \beta},\right. \\
& \left.\tau= \pm \frac{\sqrt{\alpha^{2}-4 \beta^{2}}}{4 \sqrt{2 \beta\left(N-c^{2}\right)}}, c=c\right\}, \\
& \left\{a_{0}=-\frac{\alpha}{2 \beta}, a_{1}= \pm \frac{\sqrt{\alpha^{2}-4 \beta^{2}}}{2 \beta}, b_{1}= \pm \frac{\sqrt{\alpha^{2}-4 \beta^{2}}}{8 \beta},\right. \\
& \left.\tau= \pm \frac{\sqrt{\alpha^{2}-4 \beta^{2}}}{8 \sqrt{2 \beta\left(N-c^{2}\right)}}, c=c\right\} .
\end{aligned}
$$

The first two sets gives the following wave solutions of Eq. (57):

$$
\begin{aligned}
& u_{3,5}(\tilde{x}, t)=\ln \left[-\frac{\alpha}{2 \beta} \pm \frac{\sqrt{\alpha^{2}-4 \beta^{2}}}{2 \beta}(\tanh 2 \eta)^{ \pm 1}\right], \\
& u_{2,6}(\tilde{x}, t)=\ln \left[-\frac{\alpha}{2 \beta} \pm \frac{\sqrt{\alpha^{2}-4 \beta^{2}}}{2 \beta}\left(\frac{\tanh \eta+\operatorname{coth} \eta}{2}\right)^{ \pm 1}\right],
\end{aligned}
$$


where

$$
\eta= \pm \frac{\sqrt{\alpha^{2}-4 \beta^{2}}}{4 \sqrt{2 \beta\left(N-c^{2}\right)}}\left(\sum_{j=1}^{N} x_{j}-c t\right) .
$$

The third set gives the wave solutions

$$
\begin{aligned}
u_{3,7}(\tilde{x}, t)= & \ln \left[-\frac{\alpha}{2 \beta} \pm \frac{\sqrt{\alpha^{2}-4 \beta^{2}}}{4 \beta}(\tanh 2 \eta+\operatorname{coth} 2 \eta)\right] \\
u_{3,8}(\tilde{x}, t)= & \ln \left[-\frac{\alpha}{2 \beta} \pm \frac{\sqrt{\alpha^{2}-4 \beta^{2}}}{4 \beta}\left\{\frac{(\tanh \eta+\operatorname{coth} \eta)}{2}\right.\right. \\
& \left.\left.+\frac{2}{(\tanh \eta+\operatorname{coth} \eta)}\right\}\right]
\end{aligned}
$$

where

$$
\eta= \pm \frac{\sqrt{\alpha^{2}-4 \beta^{2}}}{8 \sqrt{2 \beta\left(N-c^{2}\right)}}\left(\sum_{j=1}^{N} x_{j}-c t\right)
$$

Case (III): If we set $A=1$ and $C=4$ in Eq. (2), and solving the system of algebraic equations using Maple, we get the following sets of solutions:

$$
\begin{aligned}
& \left\{a_{0}=-\frac{\alpha}{2 \beta}, a_{1}= \pm \frac{\sqrt{4 \beta^{2}-\alpha^{2}}}{\beta}, b_{1}=0,\right. \\
& \left.\tau= \pm \frac{\sqrt{4 \beta^{2}-\alpha^{2}}}{4 \sqrt{2 \beta\left(N-c^{2}\right)}}, c=c\right\} \\
& \left\{a_{0}=-\frac{\alpha}{2 \beta}, a_{1}=0, b_{1}= \pm \frac{\sqrt{4 \beta^{2}-\alpha^{2}}}{4 \beta},\right. \\
& \left.\tau= \pm \frac{\sqrt{4 \beta^{2}-\alpha^{2}}}{4 \sqrt{2 \beta\left(N-c^{2}\right)}}, c=c\right\} \\
& \left\{a_{0}=-\frac{\alpha}{2 \beta}, a_{1}= \pm \frac{\sqrt{4 \beta^{2}-\alpha^{2}}}{2 \beta}, b_{1}=\mp \frac{\sqrt{4 \beta^{2}-\alpha^{2}}}{8 \beta},\right. \\
& \left.\tau= \pm \frac{\sqrt{4 \beta^{2}-\alpha^{2}}}{8 \sqrt{2 \beta\left(N-c^{2}\right)}}, c=c\right\}
\end{aligned}
$$


By the same calculation as above, we obtain the following wave solutions of Eq. (57) from the first two sets of equations (76) and (77)

$$
\begin{aligned}
& u_{3,9}(\tilde{x}, t)=\ln \left[-\frac{\alpha}{2 \beta} \pm \frac{\sqrt{4 \beta^{2}-\alpha^{2}}}{2 \beta}(\tan 2 \eta)^{ \pm 1}\right], \\
& u_{3,10}(\tilde{x}, t)=\ln \left[-\frac{\alpha}{2 \beta} \pm \frac{\sqrt{4 \beta^{2}-\alpha^{2}}}{2 \beta}\left(\frac{\tan \eta-\cot \eta}{2}\right)^{ \pm 1}\right],
\end{aligned}
$$

where

$$
\eta= \pm \frac{\sqrt{4 \beta^{2}-\alpha^{2}}}{4 \sqrt{2 \beta\left(N-c^{2}\right)}}\left(\sum_{j=1}^{N} x_{j}-c t\right) .
$$

Finally, the third set (78) gives the wave solutions

$$
\begin{aligned}
u_{3,11}(\tilde{x}, t)= & \ln \left[-\frac{\alpha}{2 \beta} \pm \frac{\sqrt{4 \beta^{2}-\alpha^{2}}}{4 \beta}(\tan 2 \eta-\cot 2 \eta)\right] \\
u_{3,12}(\tilde{x}, t)= & \ln \left[-\frac{\alpha}{2 \beta} \pm \frac{\sqrt{4 \beta^{2}-\alpha^{2}}}{4 \beta}\left\{\frac{(\tan \eta-\cot \eta)}{2}\right.\right. \\
& \left.\left.-\frac{2}{(\tan \eta-\cot \eta)}\right\}\right]
\end{aligned}
$$

where

$$
\eta= \pm \frac{\sqrt{4 \beta^{2}-\alpha^{2}}}{8 \sqrt{2 \beta\left(N-c^{2}\right)}}\left(\sum_{j=1}^{N} x_{j}-c t\right) .
$$

\section{Solution of $(N+1)$-dimensional sinh-cosinh-Gordon equation}

In this section, we consider the $(N+1)$-dimensional sinh-cosinh-Gordon equation of the form [21]

$$
\sum_{j=1}^{N} u_{x_{j} x_{j}}-u_{t t}-\alpha \cosh (u)-\beta \sinh (2 u)=0 .
$$


Using the transformation

$$
u\left(x_{1}, x_{2}, \cdots, x_{N}, t\right)=u(\eta), \eta=\tau\left(\sum_{j=1}^{N} x_{j}-c t\right),
$$

here $\tau \neq 0$ and $c \neq 0$ are constants, the Eq. (83) can be written as

$$
\tau^{2}\left(N-c^{2}\right) u^{\prime \prime}-\alpha \cosh (u)-\beta \sinh (2 u)=0,
$$

where the prime denotes derivative with respect to $\eta$. Next, we introduce the transformation $u=\ln v$, then we have

$$
u^{\prime \prime}=\frac{v^{\prime \prime} v-\left(v^{\prime}\right)^{2}}{v^{2}}, \quad \cosh (u)=\frac{v+v^{-1}}{2}, \quad \sinh (2 u)=\frac{v^{2}-v^{-2}}{2} .
$$

Substituting these transformations (85) in Eq. (84), we can rewrite the $(N+1)$ dimensional sinh-cosinh-Gordon Eq. (83) in the following form

$$
2 \tau^{2}\left(N-c^{2}\right)\left(v^{\prime \prime} v-\left(v^{\prime}\right)^{2}\right)-\alpha\left(v^{3}+v\right)-\beta\left(v^{4}-1\right)=0 .
$$

To determine the parameter $M$, we balance the linear terms of highest order in Eq. (86) with the highest order nonlinear terms which gives $M=1$. As a result, the modified tanh-coth method (1) admits the use of the finite expansion

$$
v(\eta)=a_{0}+a_{1} Y+\frac{b_{1}}{Y} .
$$

Substituting Eq. (87) in the reduced ODE (86) and using Eq. (2) collecting the coefficients of $Y$, yields a system of algebraic equations for $a_{0}, a_{1}, b_{1}, \tau$ and $c$.

Case (I): If we set $A=\frac{1}{2}$ and $C=-\frac{1}{2}$ in equation (2), and solving the system of algebraic equations using Maple, we obtain the following three sets of solutions:

$$
\begin{gathered}
\left\{a_{0}=-\frac{\alpha}{2 \beta}, a_{1}= \pm \frac{\sqrt{\alpha^{2}+4 \beta^{2}}}{2 \beta}, b_{1}=0,\right. \\
\left.\tau=\tau, c= \pm \frac{\sqrt{-\alpha^{2}-4 \beta^{2}+2 \tau^{2} N \beta}}{\tau \sqrt{2 \beta}}\right\}
\end{gathered}
$$




$$
\begin{aligned}
& \left\{a_{0}=-\frac{\alpha}{2 \beta}, a_{1}=0, b_{1}= \pm \frac{\sqrt{\alpha^{2}+4 \beta^{2}}}{2 \beta},\right. \\
& \left.\tau=\tau, c= \pm \frac{\sqrt{-\alpha^{2}-4 \beta^{2}+2 \tau^{2} N \beta}}{\tau \sqrt{2 \beta}}\right\}, \\
& \left\{a_{0}=-\frac{\alpha}{2 \beta}, a_{1}= \pm \frac{\sqrt{\alpha^{2}+4 \beta^{2}}}{4 \beta}, b_{1}= \pm \frac{\sqrt{\alpha^{2}+4 \beta^{2}}}{4 \beta},\right. \\
& \left.\tau=\tau, c= \pm \frac{\sqrt{-\alpha^{2}-4 \beta^{2}+8 \tau^{2} N \beta}}{2 \tau \sqrt{2 \beta}}\right\} .
\end{aligned}
$$

Substituting $Y=\tanh \eta \pm i \operatorname{sech} \eta$ and $Y=\operatorname{coth} \eta \pm \operatorname{csch} \eta$ in equation (87), the first two sets (88) and (89) gives the wave solutions

$$
\begin{aligned}
& u_{4,1}(\tilde{x}, t)=\ln \left[-\frac{\alpha}{2 \beta} \pm \frac{\sqrt{\alpha^{2}+4 \beta^{2}}}{2 \beta}(\tanh \eta \pm i \operatorname{sech} \eta)^{ \pm 1}\right] \\
& u_{4,2}(\tilde{x}, t)=\ln \left[-\frac{\alpha}{2 \beta} \pm \frac{\sqrt{\alpha^{2}+4 \beta^{2}}}{2 \beta}(\operatorname{coth} \eta \pm \operatorname{csch} \eta)^{ \pm 1}\right]
\end{aligned}
$$

where

$$
\tilde{x}=x_{1}, x_{2}, \cdots, x_{N} \quad \text { and } \quad \eta=\tau\left(\sum_{j=1}^{N} x_{j} \pm \frac{\sqrt{-\alpha^{2}-4 \beta^{2}+2 \tau^{2} N \beta}}{\tau \sqrt{2 \beta}} t\right) .
$$

Finally, the third set gives the wave solutions

$$
\begin{aligned}
u_{4,3}(\tilde{x}, t)= & \ln \left[-\frac{\alpha}{2 \beta} \pm \frac{\sqrt{\alpha^{2}+4 \beta^{2}}}{4 \beta}\{(\tanh \eta \pm i \operatorname{sech} \eta)\right. \\
& \left.\left.+\frac{1}{(\tanh \eta \pm i \operatorname{sech} \eta)}\right\}\right] \\
u_{4,4}(\tilde{x}, t)= & \ln \left[-\frac{\alpha}{2 \beta} \pm \frac{\sqrt{\alpha^{2}+4 \beta^{2}}}{4 \beta}\{(\operatorname{coth} \eta \pm \operatorname{csch} \eta)\right. \\
& \left.\left.+\frac{1}{(\operatorname{coth} \eta \pm \operatorname{csch} \eta)}\right\}\right]
\end{aligned}
$$


where

$$
\eta=\tau\left(\sum_{j=1}^{N} x_{j} \pm \frac{\sqrt{-\alpha^{2}-4 \beta^{2}+8 \tau^{2} N \beta}}{2 \tau \sqrt{2 \beta}} t\right)
$$

Case (II): If we set $A=1$ and $C=-4$ in equation (2) and by the same calculation as above, the following sets of solutions are obtained:

$$
\begin{aligned}
& \left\{a_{0}=-\frac{\alpha}{2 \beta}, a_{1}= \pm \frac{\sqrt{\alpha^{2}+4 \beta^{2}}}{\beta}, b_{1}=0,\right. \\
& \left.\tau=\tau, c= \pm \frac{\sqrt{-\alpha^{2}-4 \beta^{2}+32 \tau^{2} N \beta}}{4 \tau \sqrt{2 \beta}}\right\} \\
& \left\{a_{0}=-\frac{\alpha}{2 \beta}, a_{1}=0, b_{1}= \pm \frac{\sqrt{\alpha^{2}+4 \beta^{2}}}{4 \beta},\right. \\
& \left.\tau=\tau, c= \pm \frac{\sqrt{-\alpha^{2}-4 \beta^{2}+32 \tau^{2} N \beta}}{4 \tau \sqrt{2 \beta}}\right\} \\
& \left\{a_{0}=-\frac{\alpha}{2 \beta}, a_{1}= \pm \frac{\sqrt{\alpha^{2}+4 \beta^{2}}}{2 \beta}, b_{1}= \pm \frac{\sqrt{\alpha^{2}+4 \beta^{2}}}{8 \beta},\right. \\
& \left.\tau=\tau, c= \pm \frac{\sqrt{-\alpha^{2}-4 \beta^{2}+128 \tau^{2} N \beta}}{8 \tau \sqrt{2 \beta}}\right\} .
\end{aligned}
$$

The first two sets gives the following wave solutions of Eq. (83):

$$
\begin{aligned}
& u_{4,5}(\tilde{x}, t)=\ln \left[-\frac{\alpha}{2 \beta} \pm \frac{\sqrt{\alpha^{2}+4 \beta^{2}}}{2 \beta}(\tanh 2 \eta)^{ \pm 1}\right] \\
& u_{4,6}(\tilde{x}, t)=\ln \left[-\frac{\alpha}{2 \beta} \pm \frac{\sqrt{\alpha^{2}+4 \beta^{2}}}{2 \beta}\left(\frac{\tanh \eta+\operatorname{coth} \eta}{2}\right)^{ \pm 1}\right]
\end{aligned}
$$

where

$$
\eta=\tau\left(\sum_{j=1}^{N} x_{j} \pm \frac{\sqrt{-\alpha^{2}-4 \beta^{2}+32 \tau^{2} N \beta}}{4 \tau \sqrt{2 \beta}} t\right)
$$


The third set gives the solutions

$$
\begin{aligned}
u_{4,7}(\tilde{x}, t)= & \ln \left[-\frac{\alpha}{2 \beta} \pm \frac{\sqrt{\alpha^{2}+4 \beta^{2}}}{4 \beta}(\tanh 2 \eta+\operatorname{coth} 2 \eta)\right] \\
u_{4,8}(\tilde{x}, t)= & \ln \left[-\frac{\alpha}{2 \beta} \pm \frac{\sqrt{\alpha^{2}+4 \beta^{2}}}{4 \beta}\left\{\frac{(\tanh \eta+\operatorname{coth} \eta)}{2}\right.\right. \\
& \left.\left.+\frac{2}{(\tanh \eta+\operatorname{coth} \eta)}\right\}\right]
\end{aligned}
$$

where

$$
\eta=\tau\left(\sum_{j=1}^{N} x_{j} \pm \frac{\sqrt{-\alpha^{2}-4 \beta^{2}+128 \tau^{2} N \beta}}{8 \tau \sqrt{2 \beta}} t\right) .
$$

Case (III): If we set $A=1$ and $C=4$ in Eq. (2), and solving the system of algebraic equations using Maple, we get the following sets of solutions:

$$
\begin{aligned}
& \left\{a_{0}=-\frac{\alpha}{2 \beta}, a_{1}= \pm \frac{\sqrt{\alpha^{2}+4 \beta^{2}}}{\beta} i, b_{1}=0,\right. \\
& \left.\tau= \pm \frac{\sqrt{\alpha^{2}+4 \beta^{2}}}{4 \sqrt{2 \beta\left(c^{2}-N\right)}}, c=c\right\}, \\
& \left\{a_{0}=-\frac{\alpha}{2 \beta}, a_{1}=0, b_{1}= \pm \frac{\sqrt{\alpha^{2}+4 \beta^{2}}}{4 \beta} i,\right. \\
& \left.\tau= \pm \frac{\sqrt{\alpha^{2}+4 \beta^{2}}}{4 \sqrt{2 \beta\left(c^{2}-N\right)}}, c=c\right\}, \\
& \left\{a_{0}=-\frac{\alpha}{2 \beta}, a_{1}= \pm \frac{\sqrt{\alpha^{2}+4 \beta^{2}}}{2 \beta} i, b_{1}=\mp \frac{\sqrt{\alpha^{2}+4 \beta^{2}}}{8 \beta} i\right. \\
& \left.\tau= \pm \frac{\sqrt{\alpha^{2}+4 \beta^{2}}}{8 \sqrt{2 \beta\left(c^{2}-N\right)}}, c=c\right\} .
\end{aligned}
$$


By the same calculation as above, we obtain the following wave solutions of Eq. (83) from the first two sets of equations (102) and (103)

$$
\begin{aligned}
& u_{4,9}(\tilde{x}, t)=\ln \left[-\frac{\alpha}{2 \beta} \pm i \frac{\sqrt{\alpha^{2}+4 \beta^{2}}}{2 \beta}(\tan 2 \eta)^{ \pm 1}\right], \\
& u_{4,10}(\tilde{x}, t)=\ln \left[-\frac{\alpha}{2 \beta} \pm i \frac{\sqrt{\alpha^{2}+4 \beta^{2}}}{2 \beta}\left(\frac{\tan \eta-\cot \eta}{2}\right)^{ \pm 1}\right],
\end{aligned}
$$

where

$$
\eta= \pm \frac{\sqrt{\alpha^{2}+4 \beta^{2}}}{4 \sqrt{2 \beta\left(c^{2}-N\right)}}\left(\sum_{j=1}^{N} x_{j}-c t\right) .
$$

Finally, the third set (104) gives the wave solutions

$$
\begin{aligned}
u_{4,11}(\widetilde{x}, t)= & \ln \left[-\frac{\alpha}{2 \beta} \pm i \frac{\sqrt{\alpha^{2}+4 \beta^{2}}}{4 \beta}(\tan 2 \eta-\cot 2 \eta)\right], \\
u_{4,12}(\tilde{x}, t)= & \ln \left[-\frac{\alpha}{2 \beta} \pm i \frac{\sqrt{\alpha^{2}+4 \beta^{2}}}{4 \beta}\left\{\frac{(\tan \eta-\cot \eta)}{2}\right.\right. \\
& \left.\left.-\frac{2}{(\tan \eta-\cot \eta)}\right\}\right],
\end{aligned}
$$

where

$$
\eta= \pm \frac{\sqrt{\alpha^{2}+4 \beta^{2}}}{8 \sqrt{2 \beta\left(c^{2}-N\right)}}\left(\sum_{j=1}^{N} x_{j}-c t\right) .
$$

\section{Conclusion}

In this paper, many new travelling wave solutions for the $(N+1)$-dimensional nonlinear evolution equations is obtained by using the tanh-coth method. By using the solution of the auxiliary equation (4) in the tanh-coth function method, we obtain new solutions of the $(N+1)$-dimensional generalized Boussinesq equation, $(N+1)$-dimensional sine-cosine-Gordon equation, $(N+1)$-double sinh-Gordon equation and $(N+1)$-sinh-cosinh-Gordon equations. This tanhcoth method also suggests that one can get different exact travelling wave solutions by choosing different auxiliary equations in the tanh-function. The result 
reveals that the implemented technique is quite efficient and is practically well suited for soling the higher dimensional equations. The correctness of these solutions are ensured by testing them on computer with the aid of symbolic computation software Maple.

\section{REFERENCES}

[1] M.A. Abdou, A.A. Soliman and S.T. Basyony, New application of exp-function method for improved Boussinesq equation. Phys. Lett. A, 369 (2007), 469-475.

[2] C. Deng, New exact solutions to the Zakharov-Kuznetsov equation and its generalized form. Communications in Nonlinear Science and Numerical Simulation, 15 (2010), 857-868.

[3] M. Dehghan and F. Shakeri, Use of He's homotopy perturbation method for solving a partial differential equation arising in modeling of flow in porous media. Journal of Porous Media, 11 (2008), 765-778.

[4] M. Dehghan and J. Manafian, The solution of the variable coefficients fourth-order parabolic partial differential equations by the homotopy perturbation method. Zeitschrift fuer Naturforschung A, 64a (2009), 411-419.

[5] M. Dehghan and F. Shakeri, Application of He's variational iteration method for solving the Cauchy reaction-diffusion problem. Journal of Computational and Applied Mathematics, 214 (2008), 435-446.

[6] S.A. Elwakil, S.K. El-labany, M.A. Zahran and R. Sabry, Modified extended tanhfunction method for solving nonlinear partial differential equations. Phys. Lett. A, 299 (2002), 179-188.

[7] SA. El-Wakil, SK. El-Labany, MA. Zahran and R. Sabry, Modified extended tanh-function method and its applications to nonlinear equations. Appl. Math. Comput., 161 (2005), 403-412.

[8] E. Fan, Extended tanh-function method and its applications to nonlinear equations. Physics Letters A, 277 (2000), 212-218.

[9] E.S. Fahmy, H.A. Abdusalam and K.R. Raslan, On the solutions of the timedelayed Burgers equation. Nonlinear Analysis: Theory, Methods Applications, 69 (2008), 4775-4786.

[10] Y. Guo and S. Lai, New exact solutions for an $(N+1)$-dimensional generalized Boussinesq equation. Nonlinear Analysis: Theory, Methods Applications, 72 (2010), 2863-2873. 
[11] Z.Z. Ganji, D.D. Ganji and H. Bararnia, Approximate general and explicit solutions of nonlinear BBMB equations by Exp-function method. Applied Mathematical Modelling, 33(4) (2009), 1836-1841.

[12] J.H. He and X.H. Wu, Exp-function method for nonlinear wave equations. Chaos Solitons Fract., 30 (2006), 700-708.

[13] L. Kavitha, A. Prabhu and D. Gopi, New exact shape changing solitary solutions of a generalized Hirota equation with nonlinear inhomogeneities. Chaos Solitons and Fractals, 42 (2009), 2322-2329.

[14] J. Liu, L. Yang and K. Yang, Jacobi elliptic function solutions of some nonlinear PDEs. Physics Letters A, 325 (2004), 268-275.

[15] R. Mokhtari, Variational iteration method for solving nonlinear differentialdifference equations. Int. J. Nonlinear Sci. Numer. Simul., 9 (2008), 19-24.

[16] W. Malfliet and W. Hereman, The Tanh method: I Exact solutions of nonlinear evolution and wave equations. Phys Scripta, 54 (1996), 563-568.

[17] R. Sakthivel and C. Chun, New solitay wave solutions of some nonlinear evolution equations with distinct physical structures. Rep. Math. Phys., 62 (2008), 389-398.

[18] R. Sakthivel and C. Chun, New soliton solutions of Chaffee-Infante equations. Z. Naturforsch., 65a (2010), 197-202.

[19] J. Lee and R. Sakthivel, Exact traveling wave solutions for some nonlinear evolution equations. Communications in Mathematical Sciences, 7(4) (2009), 10531062.

[20] J. Lee and R. Sakthivel, Travelling wave solutions for $(N+1)$-dimensional nonlinear evolution equations. Pramana Journal of Physics, 45 (2010), 565-578.

[21] D.S. Wang, Z. Yan and H. Li, Some special types of solutions of a class of the $(N+1)$-dimensional nonlinear wave equations. Computers and Mathematics with Applications, 56 (2008), 1569-1579.

[22] L. Wazzan, A modified tanh-coth method for solving the general Burgers-Fisher and the Kuramoto-Sivashinsky equations. Commun. Nonlinear Sci. Numer. Simul., 14 (2009), 2642-2652.

[23] A.M. Wazwaz, Travelling wave solutions of generalized forms of Burgers, Burgers-KdV and Burgers-Huxley equations. Applied Mathematics and Computation, 169 (2005), 639-656.

[24] A.M. Wazwaz, Multiple soliton solutions and multiple singular soliton solutions for the $(3+1)$-dimensional Burgers equations. Applied Mathematics and Computation, 204 (2008), 942-948. 
[25] Z. Yan, Similarity transformations and exact solutions for a family of higherdimensional generalized Boussninesq equations. Phys. Lett. A, 361 (2007), 223230.

[26] S. Zhang and H. Zhang, Exp-function method for $N$-soliton solutions of nonlinear evolution equations in mathematical physics. Physics Letters A, 373 (30), (2009) 2501-2505.

[27] S. Zhang and H. Zhang, Variable-coefficient discrete tanh method and its application to $(2+1)$-dimensional Toda equation. Physics Letters A, 373(33) (2009), 2905-2910. 